\title{
Ambiguous Proximity Distribution
}

\author{
Quanquan Wang ${ }^{1,2^{*}}$, Yongping $\mathrm{Li}^{1}$ \\ ${ }^{1}$ Shanghai Institute of Applied Physics, Chinese Academy of Sciences, Shanghai, 201800, \\ China \\ ${ }^{2}$ University of Chinese Academy of Sciences, Bejing 100049, China \\ \{wangquanquan, ypli\}@sinap.ac.cn
}

\begin{abstract}
Proximity Distribution Kernel is an effective method for bag-offeatues based image representation. In this paper, we investigate the soft assignment of visual words to image features for proximity distribution. Visual word contribution function is proposed to model ambiguous proximity distributions. Three ambiguous proximity distributions is developed by three ambiguous contribution functions. The experiments are conducted on both classification and retrieval of medical image data sets. The results show that the performance of the proposed methods, Proximity Distribution Kernel (PDK), is better or comparable to the state-of-the-art bag-of-features based image representation methods.
\end{abstract}

Keywords: Bag-of-Features, Proximity Distribution Kernel, Soft Assignment

\section{Introduction}

Due to the rapid growth of modern digital imaging and video technologies, the volume of visual data is also increasing significantly. The management and retrieval of visual information has attracted much attention of the research community of computer vision, machine learning, and scientific computing [1-10]. Recently, Content-Based Image Retrieval (CBIR) has been one of the most popular research areas $[11-14,63,64]$. Given an query image, this problem is to retrieval images from a large image database based on the visual similarity between the query and the database images. In this case, representing the visual information of each images as some feature measures are critical [15-20]. Most often, both global and local visual information are important. Cognitive science community has shed some light on this topic, in particular in the ground laying work of Kong [21, 22] on how human visual cognition processes global and local visual information and utilize both type of information to solve complex visual problems. Kong's work on global/local visual information processing has not only made great impact in the field of cognitive science, but also inspired many advances outside cognitive science, such as in the one discussed in this paper [21, 22]. J. Yang et al. [23] presented a novel approach for foreground objects removal while ensuring structure coherence and texture consistency. The approach used structure as a guidance to complete the remaining scene. The work benefits a wide range of applications especially for the online massive collections of imagery, such as photo localization and scene reconstructions. 
Moreover, this work is applied to privacy protection by removing people from the scene. Inspired by Kong's work [21, 22], various image representation methods have been proposed. Among them, local visual descriptors has been shown to be outperforming other visual methods [24-28]. This kind of methods are also called bag-of-features since each images is represented as a collection of local features [2931].

This work presents a novel content-based image retrieval system, which is based on the Visual Words (VW) framework. VW framework is a recently introduced framework, and it has been successfully applied to scenery and object image retrieval tasks [32, 33, 15]. The VW model represents each image using a discrete and disjunct visual vocabulary which is composed of many visual word elements [34-37]. Base on this model, it is possible to split an image into a set of visual features and to represent the image using the statistics of these local visual features. The ideal intrinsic features should be transition, rotation, and scaling invariant [38, 39]. However, in many applications, it is hard to find the ideal features. In our system the visual features are the key points with SIFT (Scale Invariant Feature Transform) descriptors [40-43], or image patches (small sub images) [44-47]. One effective statistical image presentation of VM model is Proximity Distribution (PD) [48, 49]. Moreover, a corresponding kernel function is also proposed to match a pair of proximity distributions. The PD is defined as the distributions of co-occurring local features as they appear at increasing distances from one another. One inherent procedure of the PD model is the discretization of visual words from continuous image local features. In this study we investigate the effect of ambiguity modeling on ambiguous proximity model. To the best of our knowledge, there are no published studies on the application of visual word ambiguity in constructing proximity distribution. The main contribution of this paper is an investigation of visual word ambiguity leading to explicit Visual Word Contribution Function for the Ambiguous Proximity Distribution model. Moreover, we try to develop a kernel to match two Ambiguous Proximity Distribution, so that it can be used in image retrieval.

The paper is organized as follows: In section 2, we introduce the proposed Ambiguous Proximity Distribution Kernel. Section 2.1 discusses the original Proximity Distribution Kernel model. Section 2.2 introduces the Visual Word Contribution Function for generalization of hard and soft assignment of a local feature to a word in the dictionary, and by developing three ambiguous contribution functions, we proposed three Ambiguous Proximity Distributions in section 2.3. Section 3 presents the experimental results and Section 4 concludes with the final remarks.

\section{Ambiguous Proximity Distribution Kernel}

Let $X$ be an image and $\{x l\} ; l=1, \cdots \cdots, L$ a collection of $L$ local features extracted from $X$. Typically, the local features or regions $x l$ are detected interest regions with SIFT descriptors [40], or densely sampled image patches [50]. In the learning phase, we construct a codebook $V$ using a clustering algorithm. Usually, k-means is used to cluster centers of features which extracted from all images in the database, then, these cluster centers are used as a vocabulary (codebook) $V=\{v 1, \cdots \cdots, v K\}$ with $K$ visual words for all images to get word vector representation [51-53]. On the set of all 
available features of query image $X$, we perform vector-quantization, to arrive at $K$ codebook elements $V=\{v 1, \cdots \cdots, v K\}$. So, in this first coding stage, the image $X$ is represented by the local feature $\{(x l, \alpha l)\} k=1, \cdots \cdots, L$, with each $\_l$ identified with the integers $i=1, \cdots \cdots, K$.

$$
\alpha_{l}=\arg \min _{i}(\mathrm{D}(\mathrm{v} i, \mathrm{x} l))
$$

where $x l$ is image region $l$, and $D(v i, x l)$ is the distance between a codeword $v i$ and region $x l$.

Given a vocabulary of $K$ of codewords, and the traditional VW approach describes an image $X$ by a distribution over the visual words. For each word $v i$ in the vocabulary $V$, the traditional VW model estimates the distribution of visual words in an image by

$$
H_{C B}^{X}(\mathrm{i})=\frac{1}{L} \sum_{l=1}^{L} I\left(\alpha_{1}=\mathrm{i}\right)
$$

The indicator $I(x)$ outputs 1 when the Boolean variable $x$ is true and 0 otherwise, so that,

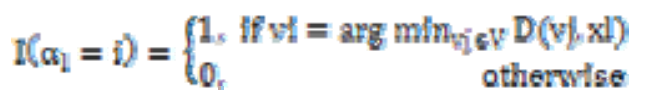

By applying $I\left(\alpha_{l}=i\right)$, the region $x l$ is only assigned to the nearest word $v i$ in the dictionary. The VW model represents an image by a histogram of word frequencies that describes the probability density over codewords.

\subsection{Proximity Distribution Kernel}

The proximity distribution kernel(PDK) is proposed by Ling and Soatto in [54], which matches distributions of co-occurring local features as they appear at increasing distances from one another in an image $X$. In this case, each feature point $x l$ is first mapped to one of $K$ discrete visual words $v i \in V$, which are the prototypical local features identified via clustering on local features of many training images.

Each local feature set $\left\{x l, \alpha_{l}\right\}, l=1, \cdots \cdots, L$ of $X$ is converted to a $K \times K \times R$ dimensional histogram. This histogram counts the number of times each visual word co-occurs within the $r=1, \cdots \cdots, R$ spatially nearest neighbor features of any other visual word. Specifically, for a given image input represented as a local feature set $X$, each histogram element $H^{X}(I, j, r)$ is defined as the number of times visual word type $j$ occurs within the $r$ spatially nearest neighbors of a visual word of type $i$. The proximity distribution of $X$ is given as 


$$
\begin{aligned}
& H_{P D}^{X}(\mathrm{i}, \mathrm{j}, \mathrm{r})=\#\left\{\left(\alpha_{1}, \alpha_{\mathrm{m}}\right): \alpha_{1}=\mathrm{i}, \alpha_{\mathrm{m}}=\mathrm{j}, \mathrm{d}_{\mathrm{NN}}\left(\mathrm{x}_{1}, \mathrm{x}_{\mathrm{m}}\right) \leq \mathrm{r}\right\} \\
& =\sum_{l=1}^{n} \sum_{m=1}^{n} I\left(\alpha_{1}=\mathrm{i}\right) I\left(\alpha_{m}=j\right) I\left(\mathrm{~d}_{\mathrm{NN}}\left(\mathrm{x}_{1}, \mathrm{x}_{\mathrm{m}}\right) \leq \mathrm{r}\right)
\end{aligned}
$$

where $d_{N N}\left(x_{l}, x_{m}\right) \leq r$ indicates that $x_{m}$ is within the $r$ - th nearest neighbors of $x_{l} . R$ is the size of the neighborhood. Since $r=1, \cdots \cdots, R$, apparently it is a cumulative distribution of the cooccurring pairs of words. The (unnormalized) proximity distribution kernel (PDK) value between two images with feature sets $Y$ and $Z$ is then

$$
K_{P D K}(\mathrm{Y}, \mathrm{Z})=\sum_{i=1}^{K} \sum_{j=1}^{K} \sum_{r=1}^{R} \min \left(\mathrm{H}^{\mathrm{Y}}(\mathrm{i}, \mathrm{j}, \mathrm{r}), \mathrm{H}^{\mathrm{Z}}(\mathrm{i}, \mathrm{j}, \mathrm{r})\right)
$$

where $H_{Y}$ and $H_{Z}$ are the associated arrays of histograms computed for the two input feature sets for image $Y$ and $Z$.

\subsection{Visual Word Contribution Function}

In this section, we generalize the bag-of-features based image presentation as a visual word contribution form. First of all, for a region $x_{l}$ in an image $X$, we define it's contribution in the constructing of an statistical presentation (histogram or proximity distribution) as Visual Word Contribution Function. In detail, in the vector quantization phase, the region $x_{l}$ will only be assigned to the nearest visual word $v_{i}$ in the dictionary $V$ as $\alpha_{i}=\arg \min _{k}\left(D\left(v_{k}, x_{i}\right)\right)$ in (2), while not considering any other visual words $\left\{v j \mid\left(D\left(v_{j}, x_{i}\right)\right)>D\left(v_{k}, x_{i}\right) ; j \neq k\right\}$. When constructing the traditional VW based histogram $H_{C B}$ in the accumulative way in (2), the quantities of $x_{l}$ 's contribution in accumulating the $i$-th bin of the histogram $H_{C B}(i)$ is $I\left(\alpha_{l}=i\right)$ as (3).

Here, we define the Visual Word Contribution Function of $x_{l}$ for $v_{i}$ as its contribution in accumulating the $i$-th bin as $F\left(v_{i}, x_{l}\right)$, so that for coodbook base histogram can be rewritten as

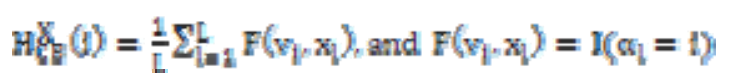

Then we consider the Visual Word Contribution for Proximity Distribution $\mathrm{H}_{\mathrm{PD}}$. Apparently, by $\mathbb{F}\left(v_{i}, x_{l}\right)=\mathbb{\Perp}\left(a_{1}=\right.$, (4) can be rewritten as

$$
H_{P D}^{X}(\mathrm{i}, \mathrm{j}, \mathrm{r})=\sum_{l=1}^{n} \sum_{m=1}^{n} F\left(\mathrm{v}_{\mathrm{i}}, \mathrm{x}_{\mathrm{l}}\right) F\left(\mathrm{v}_{j}, \mathrm{x}_{m}\right) I\left(\mathrm{~d}_{\mathrm{NN}}\left(\mathrm{x}_{1}, \mathrm{x}_{\mathrm{m}}\right) \leq \mathrm{r}\right)
$$

In the above histogram and proximity distribution, one inherent component of the hard assignment for codebook model is the assignment of image local feature to visual words in the vocabulary by using Hard Assignment Contribution Function as $\mathrm{F}_{\text {hard }}\left(\mathrm{V}_{\mathrm{i}}, \mathrm{X}_{\mathrm{l}}\right)$ 


$$
F_{\text {Lard }}\left(v_{l}, x_{1}\right)=\left\{\begin{array}{lr}
1, & \text { if } \Phi_{l=1} \\
0, & \text { otherwise }
\end{array}\right.
$$

Here, an important assumption is that a discrete visual word is a characteristics representative of a continuous image feature.

\subsection{Ambiguous Proximity Distribution}

The continuous nature of local visual features indicates selecting a most representative visual word from a visual vocabulary. It is possible that one local feature can have zero, one, or multiple optimal visual words in a visual vocabulary [34]. If there is only one optimal visual word, there is no ambiguity. We propose a robust alternative method of discrete proximity distribution to estimate a probability density function, which is based on kernel density estimation [34].We call it Ambiguous Proximity Distribution. By giving three different ambiguous contribution function to replace the hard contribution function $F_{\text {hard }}$, we developed three kinds of Ambiguous Proximity

Distributions (APD) as follows:

Kernel Proximity Distribution In the VW model, the histogram estimation function (presented by hard contribution function Fhard) of the visual words may be replaced by a kernel density estimation function. Moreover, a suitable kernel (such as the Gaussian kernel $K_{\sigma}(\mathrm{x})=\frac{1}{\sqrt{2 \pi} \sigma} \exp \left(-\frac{1}{2} \frac{x^{2}}{\sigma^{2}}\right)$, where $\sigma$ is the bandwidth parameter of kernel $\left.K_{\sigma}(\mathrm{x})\right)$ is used, and it allows kernel density estimation to become a part of the visual word. With this function, the Kernel Proximity Distribution is given as,

$$
\begin{aligned}
& H_{\mathrm{ker}}^{X}(\mathrm{i}, \mathrm{j}, \mathrm{r})=\sum_{l=1}^{L} \sum_{m=1}^{L}\left\{F_{\mathrm{ker}}\left(\mathrm{v}_{\mathrm{i}}, \mathrm{x}_{\mathrm{l}}\right) F_{\mathrm{ker}}\left(\mathrm{v}_{j}, \mathrm{x}_{m}\right) I\left(\mathrm{~d}_{\mathrm{NN}}\left(\mathrm{x}_{\mathrm{l}}, \mathrm{x}_{\mathrm{m}}\right) \leq \mathrm{r}\right)\right\} \\
& =\sum_{l=1}^{L} \sum_{m=1}^{L}\left\{K_{\sigma}\left(\mathrm{D}\left(\mathrm{v}_{\mathrm{i}}, \mathrm{x}_{1}\right)\right) K_{\sigma}\left(\mathrm{D}\left(\mathrm{v}_{j}, \mathrm{x}_{m}\right)\right) I\left(\mathrm{~d}_{\mathrm{NN}}\left(\mathrm{x}_{1}, \mathrm{x}_{\mathrm{m}}\right) \leq \mathrm{r}\right)\right\}
\end{aligned}
$$

where $F_{\text {ker }}\left(\mathrm{v}_{\mathrm{i}}, \mathrm{X}_{1}\right)$ is the Kernel Contribution Function, as follows,

$$
F_{\text {ker }}\left(\mathrm{v}_{\mathrm{i}}, \mathrm{x}_{\mathrm{l}}\right)=K_{\sigma}\left(\mathrm{D}\left(\mathrm{v}_{\mathrm{i}}, \mathrm{x}_{1}\right)\right)=\frac{1}{\sqrt{2 \pi} \sigma} \exp \left(-\frac{1}{2} \frac{\mathrm{D}\left(\mathrm{v}_{\mathrm{i}}, \mathrm{x}_{1}\right)^{2}}{\sigma^{2}}\right)
$$

In this way, a kernel visual vocabulary replaces the hard mapping of local features in an image region to the visual vocabulary. This soft assignment models is realized by replacing the hard contribution function with the kernel contribution function. This soft assignment models two types of ambiguity between visual words: codeword 
uncertainty and codeword plausibility, which are introduced as follows.

Uncertainty Proximity Distribution Uncertainty Contribution Function indicates that one image local feature $\mathrm{x}_{\mathrm{l}}$ may be assigned to more than one visual words. Uncertainty Contribution Function is defined as follows,

$$
\mathrm{F}_{\text {unc }}\left(\mathrm{v}_{\mathrm{i}}, \mathrm{x}_{\mathrm{l}}\right)=\frac{K_{\sigma}\left(\mathrm{D}\left(\mathrm{v}_{\mathrm{i}}, \mathrm{x}_{\mathrm{l}}\right)\right)}{\sum_{j=1}^{|\mathrm{V}|} K_{\sigma}\left(\mathrm{D}\left(\mathrm{v}_{j}, \mathrm{x}_{\mathrm{l}}\right)\right)}
$$

$F_{u n c}$ normalizes the probabilities to 1 and is distributed over all visual words. By using $F_{u n c}$, we can define the Uncertainty Proximity Distribution as

$$
\begin{aligned}
& H_{u n c}^{X}(\mathrm{i}, \mathrm{j}, \mathrm{r})=\sum_{l=1}^{L} \sum_{m=1}^{L}\left\{K_{u n c}\left(w_{\mathrm{i}}, \mathrm{x}_{1}\right) K_{u n c}\left(w_{j}, \mathrm{x}_{m}\right) I\left(\mathrm{~d}_{\mathrm{NN}}\left(\mathrm{x}_{1}, \mathrm{x}_{\mathrm{m}}\right) \leq \mathrm{r}\right)\right\} \\
& =\sum_{l=1}^{L} \sum_{m=1}^{L}\left\{\frac{K_{\sigma}\left(\mathrm{D}\left(\mathrm{v}_{\mathrm{i}}, \mathrm{x}_{1}\right)\right)}{\sum_{k=1}^{|\mathrm{V}|} K_{\sigma}\left(\mathrm{D}\left(\mathrm{v}_{k}, \mathrm{x}_{1}\right)\right)} \frac{K_{\sigma}\left(\mathrm{D}\left(\mathrm{v}_{j}, \mathrm{x}_{1}\right)\right)}{\sum_{k=1}^{|\mathrm{V}|} K_{\sigma}\left(\mathrm{D}\left(\mathrm{v}_{k}, \mathrm{x}_{1}\right)\right)} I\left(\mathrm{~d}_{\mathrm{NN}}\left(\mathrm{x}_{1}, \mathrm{x}_{\mathrm{m}}\right) \leq \mathrm{r}\right)\right\}
\end{aligned}
$$

In this way, visual word uncertainty is kept and it has the ability to assign one local feature to multiple visual words. However, it does not take the plausibility of a visual word into account.

Plausibility Proximity Distribution Visual word Plausibility Contribution Function is proposed by by using ambiguous contribution functions. This function indicates that an image local feature may not be close enough to be represented by all the visual words in the vocabulary. Plausibility Contribution Function is defined as

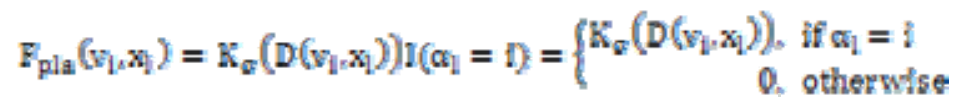

$F_{p l a}$ selects for an image local feature $x_{l}$ only the closet visual word $v_{i}$ and assigns it probability to the kernel value of that visual word. But it should be noted that it cannot select multiple visual word candidates.

In this way, we redefine the Proximity Distribution using Plausibility Contribution Function $F_{\text {pla }}$, resulting the Plausibility Proximity Distribution as

$$
\begin{aligned}
& H_{p l a}^{X}(\mathrm{i}, \mathrm{j}, \mathrm{r})=\sum_{l=1}^{L} \sum_{m=1}^{L}\left\{F_{p l a}\left(\mathrm{v}_{\mathrm{i}}, \mathrm{x}_{1}\right) F_{p l a}\left(\mathrm{v}_{j}, \mathrm{x}_{m}\right) I\left(\mathrm{~d}_{\mathrm{NN}}\left(\mathrm{x}_{\mathrm{l}}, \mathrm{x}_{\mathrm{m}}\right) \leq \mathrm{r}\right)\right\} \\
& =\sum_{l=1}^{L} \sum_{m=1}^{L}\left\{K_{\sigma}\left(\mathrm{D}\left(\mathrm{v}_{\mathrm{i}}, \mathrm{x}_{\mathrm{l}}\right)\right) I\left(\alpha_{1}=\mathrm{i}\right) K_{\sigma}\left(\mathrm{D}\left(\mathrm{v}_{j}, \mathrm{x}_{m}\right)\right) I\left(\alpha_{m}=j\right) I\left(\mathrm{~d}_{\mathrm{NN}}\left(\mathrm{x}_{1}, \mathrm{x}_{\mathrm{m}}\right) \leq \mathrm{r}\right)\right\}
\end{aligned}
$$


An unified formula is given as follows for these three ambiguous proximity distributions:

$$
\mathrm{H}^{\mathrm{X}}(\mathrm{i}, \mathrm{j}, \mathrm{r})=\sum_{l=1}^{L} \sum_{m=1}^{L}\left\{F\left(w_{\mathrm{i}}, \mathrm{x}_{1}\right) F_{p l a}\left(w_{j}, \mathrm{x}_{m}\right) I\left(\mathrm{~d}_{\mathrm{NN}}\left(\mathrm{x}_{1}, \mathrm{x}_{\mathrm{m}}\right) \leq \mathrm{r}\right)\right\}
$$

where $F\left(v_{i}, x_{l}\right)$ is to the a version of $K_{k e r}\left(v_{i}, x_{l}\right)$ for Kernel Proximity Distribution, $F_{\text {unc }}\left(v_{i}, x_{l}\right)$ for Uncertainty Proximity Distribution, or $F_{p l a}\left(v_{i}, x_{l}\right)$ for Plausibility Proximity Distribution respectively. Moreover, by setting $F\left(v_{i}, x_{l}\right)=F_{\text {hard }}\left(v_{i}, x_{l}\right)=I\left(\alpha_{l}\right.$ $=i$ ), the original hard assignment of Proximity Distribution in (4) can also be included in (15).

Then the corresponding (unnormalized) Ambiguous Proximity Distribution Kernel (APDK) value between two images with feature sets $Y$ and $Z$ is

$$
\mathrm{K}_{\text {apd }}(\mathrm{Y}, \mathrm{Z})=\sum_{i=1}^{K} \sum_{j=1}^{K} \sum_{r=1}^{R} \min \left(\mathrm{H}_{a p d}^{Y}(\mathrm{i}, \mathrm{j}, \mathrm{r}), \mathrm{H}_{\text {apd }}^{Z}(\mathrm{i}, \mathrm{j}, \mathrm{r})\right)
$$

where $\mathrm{H}_{\text {apd }}^{Y}$ and $\mathrm{H}_{\text {apd }}^{Z}$ are the associated arrays of Ambiguous Proximity Distribution computed for the two input feature sets.

\section{Experiments}

The proposed method is in general applicable for CBIR of large image databases according to learned local visual vocabulary. In this section, we demonstrate our algorithm using image patch exemplars [55] applied to a nearest neighbor classification problem medical image retrieval. We carry the experiments based on ImageClef 2007 medical image classification and ImageClef 2008 large-scale medical image retrieval competitions. In these two groups of experiments, each image is treated as a collection of image local image features.

\subsection{Experiment on ImageClef 2007 Dataset}

In the ImageClef 2007 medical image classification competition [56], a database of 12,000 categorized radiograph images is used. In this experiment, we also use this database. A set of 11,000 images are used as training set, and the remaining 1000 images are used as test images. There are 116 different classes within this database, based on the differences of either the examined region, the image orientation with respect to the body or the biological system under evaluation.

We represent each image as a bag of small patches, which are the representation of local features of an image. We extract a small patch around every pixel, using a patch size of $9 \times 9$ pixels. and the level of noise, we have also applied the Principal Component Analysis (PCA) [57, 58] procedure to the data dimensionality of descriptor vector of each local feature from 81 to 7 . The next step of our system is to learn a vocabulary of visual words based on a set of local features of images. The 
main step in the vocabulary construction procedure is clustering the patches, and we use the the k-means algorithm. A small-size vocabulary of visual words are generated. Using the generated visual vocabulary, each image $X$ is represented as an Ambiguous Proximity

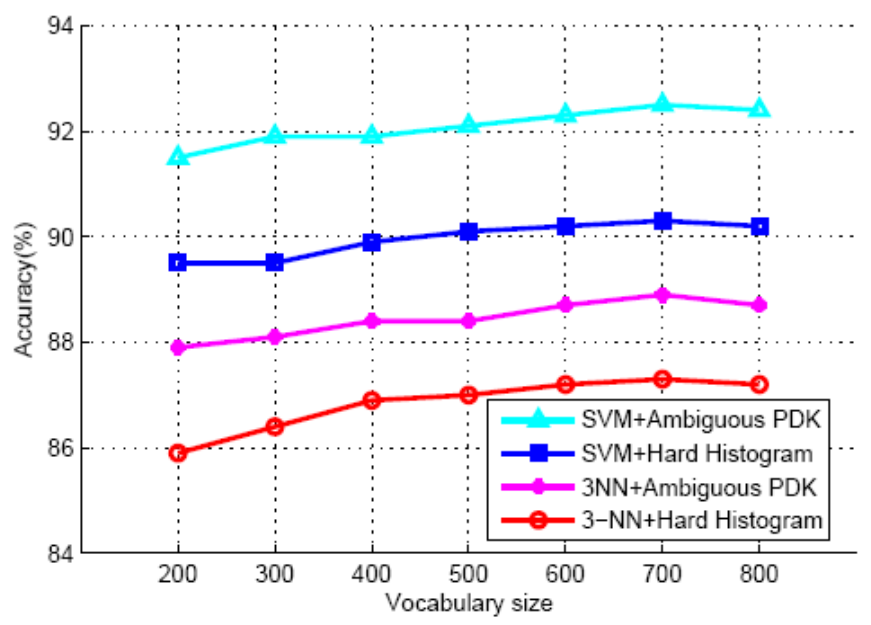

(a)

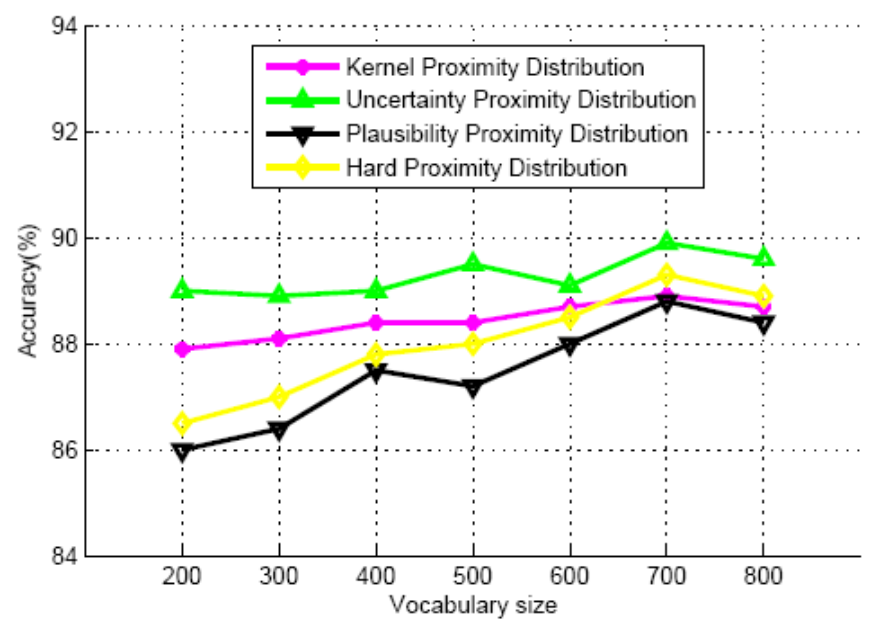

(b) 
Fig. 1 (a) Effect of vocabulary size, for K-NN and SVM classifiers. (b) Classification performance results of various types of Ambiguous PDK for the ImageClef 2007 dataset over various vocabulary sizes

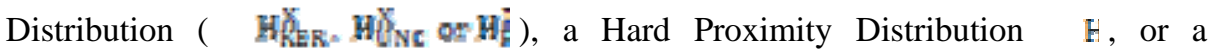
histogram of visual words. In this step images are sampled with a dense grid. We firstly use the $k$ nearest neighbor classifier for the classification problem [59-61]. Given a query image the retrieval is based on finding the nearest image in the labeled training set. With our Ambiguous Proximity Distribution, we adopt the Ambiguous Proximity Distribution Kernel $K A P D$ to compute the similarity of the Ambiguous Proximity Distribution of two patches collections for images. We use the $L_{1}$ norm distance $D^{L 1}\left(H^{X}, H^{Y}\right)$ between the word histograms of the two images $X$ and $Y$ as distance measure for hard histogram,

$$
D^{L 1}\left(\mathrm{H}^{\mathrm{X}}, \mathrm{H}^{\mathrm{Y}}\right)=\sum_{k=1}^{K}\left|\mathrm{~h}_{k}^{X}-\mathrm{h}_{k}^{Y}\right|
$$

We have found that we gain much better results using a multi-class SVM classifier. The multiclass SVM is implemented as a series of one-vs-one binary SVMs with a Ambiguous Proximity Distribution Kernel $K_{A P D}$. We run 20 cross-validation experiments trained on 10000 training images and then test it on 1000 randomly selected test images.

As Figure 1 shows, we increase the number of visual words up to 700 words. The performance of vocabulary size of 700 visual words is significantly better than that of the 200 vocabulary for the Hard Histogram baseline. However, with Ambiguous PDK (Ambiguous Proximity Distribution Kernel) the results are very similar for both sizes, as Ambiguous PDK can utilize more information of provided by vocabulary by Ambiguous Visual Word Contribution Function. Figure 1 (a) also demonstrates that using an SVM classifier provides results that are more than 3\% higher than the best K-NN classifier for both Hard Histogram baseline and Ambiguous PDK. We then make a concrete analysis of the results in Fig. 1 (b) with the various types of Ambiguous Proximity Distributions. The results show that Uncertainty Proximity Distributions $H_{U N C}$ consistently outperforms other types of ambiguity for all vocabulary sizes. But we should note that this performance gain is not always that significant. Moreover, for a vocabulary size of 200, Uncertainty Proximity Distributions $H_{U N C}$ outperforms Hard Assignment based Proximity Distributions. On the other end of the performance scale, there is Plausibility Proximity Distribution $H_{P L A}$, which always yields the lowest results. We can also see that a Kernel Proximity Distribution $H_{K E R}$, outperforms Hard Proximity Distributions for smaller vocabulary sizes.

\subsection{Large Scale Image Retrieval Experiment}

In ImageClef 2008 a large-scale medical image retrieval competition was conducted. In this competition, there are 66,000 images in the database, and also 30 query topics. Moreover, each topic is composed of one or more images and also a short textual 
description. The objective of retrieval is to return a set of 1000 images from the given database. Figure 2 shows the scores of our Ambiguous Proximity Distribution Kernel (Ambiguous PDK), along with visual retrieval algorithms submitted by additional groups [62]. As the results shown in Fig. 2, The Ambiguous PDK for either original method shows the best possible performance, and again as we decrease the returned images, we can expect more accurate results at the cost of fewer returned images.

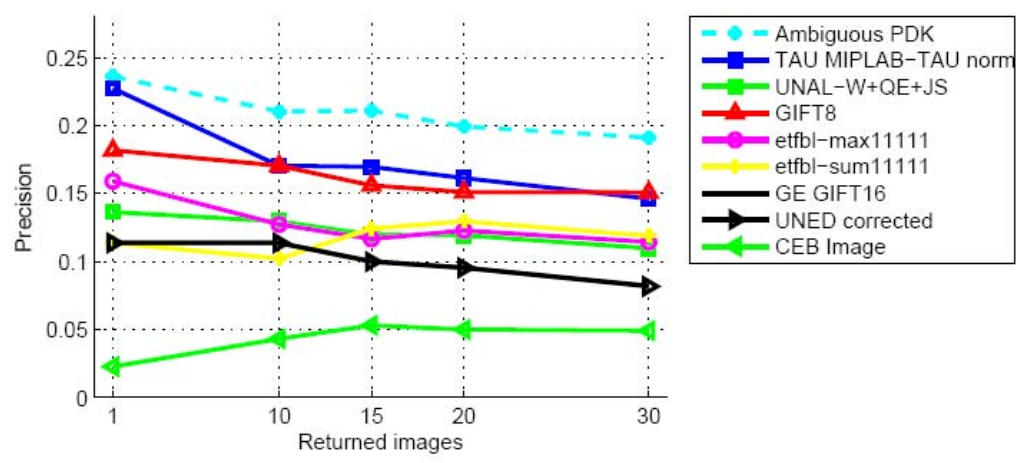

Fig. 2 Precision vs Recall graph of visual retrieval systems; ImageClef 2008 medical database. Predication shown for first 5,10,15,20 and 30 returned images.

\section{Conclusions}

This paper proposed Ambiguous Proximity Distribution, a novel image presentation combining the advantage of visual word ambiguity and ambiguous proximity distribution. A visual word contribution function framework was used for analyzing its relation with the popular VW model and developing three novel Ambiguous Proximity Distributions. An extensive comparative medical image retrieval and classification experimental analysis with state-of-the art medical image retrieval methods provided empirical evidence of the effectiveness of the proposed technique for enhancing the performance of medical image retrieval system.

\section{References}

1. Penatti, O., Silva, F., Valle, E., Gouet-Brunet, V., Torres, R.: Visual word spatial arrangement for image retrieval and classification. Pattern Recognition 47(2) (2014) 705720

2. Chen, J., Feng, B., Xu, B.: Spatial similarity measure of visual phrases for image retrieval. Lecture Notes in Computer Science (including subseries Lecture Notes in Artificial Intelligence and Lecture Notes in Bioinformatics) 8326 LNCS(PART 2) (2014) 275-282 
3. Furuya, T., Ohbuchi, R.: Visual saliency weighting and cross-domain manifold ranking for sketchbased image retrieval. Lecture Notes in Computer Science (including subseries Lecture Notes in Artificial Intelligence and Lecture Notes in Bioinformatics) 8325 LNCS(PART 1) (2014) 37-49

4. Sun, Q., Hu, F., Hao, Q.: Mobile target scenario recognition via low-cost pyroelectric sensing system: Toward a context-enhanced accurate identification. Systems, Man, and Cybernetics: Systems, IEEE Transactions on 44(3) (March 2014) 375-384

5. Sun, Q., Hu, F., Hao, Q.: Context awareness emergence for distributed binary pyroelectric sensors. In: Multisensor Fusion and Integration for Intelligent Systems (MFI), 2010 IEEE Conference on, IEEE (2010) 162-167

6. Wang, Y., Jiang, W., Agrawal, G.: Scimate: A novel mapreduce-like framework for multiple scientific data formats. In: Cluster, Cloud and Grid Computing (CCGrid), 2012 $12^{\text {th }}$ IEEE/ACM International Symposium on, IEEE (2012) 443-450

7. Wang, Y., Su, Y., Agrawal, G.: Supporting a light-weight data management layer over hdf5. In: Cluster, Cloud and Grid Computing (CCGrid), 2013 13th IEEE/ACM International Symposium on, IEEE (2013) 335-342

8. Su, Y., Wang, Y., Agrawal, G., Kettimuthu, R.: Sdquery dsi: integrating data management support with a wide area data transfer protocol. In: Proceedings of SC13: International Conference for High Performance Computing, Networking, Storage and Analysis, ACM (2013) 47

9. Xu, L., Zhan, Z., Xu, S., Ye, K.: Cross-layer detection of malicious websites. In: CODASPY. (2013) 141-152

10. Cui, S., Soh, Y.C.: Linearity indices and linearity improvement of 2-d tetralateral positionsensitive detector. Electron Devices, IEEE Transactions on 57(9) (Sept 2010) 2310-2316

11. Xia, Y., Wan, S., Yue, L.: A new texture direction feature descriptor and its application in contentbased image retrieval. Lecture Notes in Electrical Engineering 278 LNEE (2014) 143-151

12. Xia, Y., Wan, S., Yue, L.: Local spatial binary pattern: A new feature descriptor for content-based image retrieval. In: Proceedings of SPIE - The International Society for Optical Engineering. Volume 9069. (2014)

13. Guimar"aes Pedronette, D., Almeida, J., Da S. Torres, R.: A scalable re-ranking method for contentbased image retrieval. Information Sciences 265 (2014) 91-104

14. Xie, B., Mu, Y., Song, M., Tao, D.: Random projection tree and multiview embedding for largescale image retrieval. In: Neural Information Processing. Models and Applications. Springer (2010) 641-649

15. Qian, J., Yang, J., Zhang, N., Yang, Z.: Histogram of visual words based on locally adaptive regression kernels descriptors for image feature extraction. Neurocomputing 129 (2014) 516-527

16. Zhang, C., Liu, R., Qiu, T., Su, Z.: Robust visual tracking via incremental low-rank features learning. Neurocomputing 131 (2014) 237-247

17. Wu, F., Pai, H.T., Yan, Y.F., Chuang, J.: Clustering results of image searches by annotations and visual features. Telematics and Informatics 31(3) (2014) 477-491

18. Zhou, Y., Li, L., Zhao, T., Zhang, H.: Region-based high-level semantics extraction with cedd. In: Network Infrastructure and Digital Content, 2010 2nd IEEE International Conference on, IEEE (2010) 404-408

19. Zhou, Y., Li, L., Zhang, H.: Adaptive learning of region-based plsa model for total scene annotation. arXiv preprint arXiv:1311.5590 (2013) 
20. Li, X., Gao, J., Li, H., Yang, L., Srihari, R.K.: A multimodal framework for unsupervised feature fusion. In: Proceedings of the 22nd ACM international conference on Conference on information \& knowledge management, ACM (2013) 897-902

21. Kong, X., Schunn, C.D.: Global vs. local information processing in visual/spatial problem solving: The case of traveling salesman problem. Cognitive Systems Research 8(3) (2007) 192-207

22. Kong, X., Schunn, C.D., Wallstrom, G.L.: High regularities in eye-movement patterns reveal the dynamics of the visual working memory allocation mechanism. Cognitive science 34(2) (2010) 322- 337

23. Yang, J., Wang, Y., Wang, H., K., H., Wang, W., , J., S.: Automatic objects removal for scene completion. In: he 33rd Annual IEEE International Conference on Computer Communications (INFOCOM'14), Workshop on Security and Privacy in Big Data. (2014)

24. Cho, W., Seo, S., Na, I.S., Kang, S.: Automatic images classification using hdp-gmm and local image features. Lecture Notes in Electrical Engineering 280 LNEE (2014) 323-333

25. Javed, U., Riaz, M., Ghafoor, A., Ali, S., Cheema, T.: Mri and pet image fusion using fuzzy logic and image local features. The Scientific World Journal 2014 (2014)

26. Yang, F., Xu, Y.Y.,Wang, S.T., Shen, H.B.: Image-based classification of protein subcellular location patterns in human reproductive tissue by ensemble learning global and local features. Neurocomputing 131 (2014) 113-123

27. Sun, Q.,Wu, P.,Wu, Y., Guo, M., Lu, J.: Unsupervised multi-level non-negative matrix factorization model: Binary data case. Journal of Information Security 3(4) (2012)

28. Mu, Y., Ding, W., Tao, D., Stepinski, T.F.: Biologically inspired model for crater detection. In: Neural Networks (IJCNN), The 2011 International Joint Conference on, IEEE (2011) 2487-2494

29. Pinto, P., Tome, A., Santos, V.: Visual detection of vehicles using a bag-of-features approach. In: Proceedings of the 2013 13th International Conference on Autonomous Robot Systems, ROBOTICA 2013. (2013)

30. Kranthi Kiran, M., ShyamVamsi, T.: Hand gesture detection and recognition using affineshift, bag-of-features and extreme learning machine techniques. Advances in Intelligent Systems and Computing 247 (2014) 181-187

31. Yu, J., Jeon, M., Pedrycz, W.: Weighted feature trajectories and concatenated bag-offeatures for action recognition. Neurocomputing 131 (2014) 200-207

32. Zagoris, K., Pratikakis, I., Antonacopoulos, A., Gatos, B., Papamarkos, N.: Distinction between handwritten and machine-printed text based on the bag of visual words model. Pattern Recognition 47(3) (2014) 1051-1062

33. Fabian, J., Pires, R., Rocha, A.: Visual words dictionaries and fusion techniques for searching people through textual and visual attributes. Pattern Recognition Letters 39(1) (2014) 74-84

34. van Gemert, Jan C. Veenman, C.J.S.A.W.G.J.M.: Visual word ambiguity. IEEE Transactions on Pattern Analysis and Machine Intelligence 32(7) (2010) 1271 - 1283

35. Fiel, S., Sablatnig, R.: Writer identification and writer retrieval using the fisher vector on visual vocabularies. In: Proceedings of the International Conference on Document Analysis and Recognition, ICDAR. (2013) 545-549

36. Bolovinou, A., Kotsiourou, C., Amditis, A.: Dynamic road scene classification: Combining motion with a visual vocabulary model. In: Proceedings of the 16th International Conference on Information Fusion, FUSION 2013. (2013) 1151-1158

37. Wang, L., Elyan, E., Song, D.: Rebuilding visual vocabulary via spatial-temporal context similarity for video retrieval. Lecture Notes in Computer Science (including subseries 
Lecture Notes in Artificial Intelligence and Lecture Notes in Bioinformatics) 8325 LNCS(PART 1) (2014) 74-85

38. Sun, Q., Ma, R., Hao, Q., Hu, F.: Space encoding based human activity modeling and situation perception. In: Cognitive Methods in Situation Awareness and Decision Support (CogSIMA), 2013 IEEE International Multi-Disciplinary Conference on, IEEE (2013) 183-186

39. Sun, Q., Hu, F., Hao, Q.: Human activity modeling and situation perception based on fiber-optic sensing system. IEEE Transactions on Human Machine Systems (2014)

40. Sun, T., Ding, S., Xu, X.: No-reference image quality assessment through sift intensity. Applied Mathematics and Information Sciences 8(4) (2014) 1925-1934

41. Meng, X., Yin, Y., Yang, G., Xi, X.: Retinal identification based on an improved circular gabor filter and scale invariant feature transform. Sensors (Basel, Switzerland) 13(7) (2013) 9248-9266

42. Travieso, C., Del Pozo-Banos, M., Alonso, J.: Fused intra-bimodal face verification approach based on scale-invariant feature transform and a vocabulary tree. Pattern Recognition Letters 36(1) (2014) 254-260

43. Li, Y., Liu, W., Li, X., Huang, Q., Li, X.: Ga-sift: A new scale invariant feature transform for multispectral image using geometric algebra. Information Sciences (2014)

44. Makar, M., Chang, C.L., Chen, D., Tsai, S., Girod, B.: Compression of image patches for local feature extraction. In: ICASSP, IEEE International Conference on Acoustics, Speech and Signal Processing - Proceedings. (2009) 821-824

45. Shih, H.C., Chuang, C.Y., Huang, C.L., Lin, C.H.: Gender classification using bayesian classifier with local binary patch features. In: IEEE 4th International Conference on Nonlinear Science and Complexity, NSC 2012 - Proceedings. (2012) 45-50

46. Zhang, Q., Zhang, L., Yang, Y., Tian, Y., Weng, L.: Local patch discriminative metric learning for hyperspectral image feature extraction. IEEE Geoscience and Remote Sensing Letters (2013)

47. Chen, G., Sun, R., Ren, Z., Wang, Z., Sun, L.: Local shape patch based feature matching method. Zhongnan Daxue Xuebao (Ziran Kexue Ban)/Journal of Central South University (Science and Technology) 44(SUPPL.2) (2013) 33-39

48. Chandler, D., Field, D.: Estimates of the information content and dimensionality of natural scenes from proximity distributions. Journal of the Optical Society of America A: Optics and Image Science, and Vision 24(4) (2007) 922-941

49. Ling, H., Soatto, S.: Proximity distribution kernels for geometric context in category recognition. (2007)

50. Deselaers, T; Keysers, D.N.H.: Features for image retrieval: an experimental comparison. INFORMATION RETRIEVAL 11(2) (2008) 77-107

51. Ay, M., Kisi, O.: Modelling of chemical oxygen demand by using anns, anfis and k-means clustering techniques. Journal of Hydrology 511 (2014) 279-289

52. Wang, L., Pan, C.: Robust level set image segmentation via a local correntropy-based kmeans clustering. Pattern Recognition 47(5) (2014) 1917-1925

53. Lin, C.H., Chen, C.C., Lee, H.L., Liao, J.R.: Fast k-means algorithm based on a level histogram for image retrieval. Expert Systems with Applications 41(7) (2014) 3276-3283

54. Ling, HB; Soatto, S.: Proximity distribution kernels for geometric context in category recognition. 2007 IEEE 11TH INTERNATIONAL CONFERENCE ON COMPUTER VISION 1-6 (2007) 245-252

55. Varma, M., Zisserman, A.: A statistical approach to material classification using image patch exemplars. IEEE Transactions On Pattern Analysis And Machine Intelligence 31(11) (2009) 2032-2047 
56. Deselaers, T., Hanbury, A., Viitaniemi, V., Benczr, A., Brendel, M., Darczy, B., Escalante Balderas, H., Gevers, T., Hernndez Gracidas, C., Hoi, S., Laaksonen, J., Li, M., Marn Castro, H., Ney, H., Rui, X., Sebe, N., Stttinger, J., Wu, L.: Overview of the imageclef 2007 object retrieval task. Lecture Notes in Computer Science (including subseries Lecture Notes in Artificial Intelligence and Lecture Notes in Bioinformatics) 5152 LNCS (2008) 445-471

57. Li, M., Li, Y., Huang, X., Zhao, G., Tian, W.: Evaluating growth models of pseudomonas spp. in seasoned prepared chicken stored at different temperatures by the principal component analysis (pca). Food Microbiology 40 (2014) 41-47

58. Lu, Y., Gao, B., Chen, P., Charles, D., Yu, L.: Characterisation of organic and conventional sweet basil leaves using chromatographic and flow-injection mass spectrometric (fims) fingerprints combined with principal component analysis. Food Chemistry 154 (2014) 262-268

59. Niu, Y., Wang, X.: On the k-nearest neighbor classifier with locally structural consistency. Lecture Notes in Electrical Engineering 271 LNEE(VOL. 2) (2014) 269-277

60. Souza, R., Rittner, L., Lotufo, R.: A comparison between k-optimum path forest and knearest neighbors supervised classifiers. Pattern Recognition Letters 39(1) (2014) 2-10

61. Li, C.L., Wang, E., Huang, G.J., Chen, A.: Top-n query processing in spatial databases considering bi-chromatic reverse k-nearest neighbors. Information Systems 42 (2014) 123-138

62. M“uller, H., Kalpathy-Cramer, J., Kahn Jr., C., Hatt, W., Bedrick, S., Hersh, W.: Overview of the imageclefmed 2008 medical image retrieval task. Lecture Notes in Computer Science (including subseries Lecture Notes in Artificial Intelligence and Lecture Notes in Bioinformatics) 5706 LNCS (2009) 512-522

63. Shen, J., Su, P.-C., Cheung, S.-C., Zhao, J.: Virtual Mirror Rendering with Stationary RGB-D Cameras and Stored 3D Background. IEEE Transactions on Image Processing 22 (9) (2013) 1-16

64. Shen, J., \& Cheung, S. C. S. (2013, June). Layer Depth Denoising and Completion for Structured-Light RGB-D Cameras. In Computer Vision and Pattern Recognition (CVPR), 2013 IEEE Conference on (pp. 1187-1194). IEEE. 